\title{
In vitro controlled release of cisplatin from gold- carbon nanobottles via cleavable linkages
}

\author{
This article was published in the following Dove Press journal: \\ International Journal of Nanomedicine \\ 15 December 2015 \\ Number of times this article has been viewed
}

Jian $\mathrm{Li}^{\prime}$

Sia Lee Yoong ${ }^{2}$

Wei Jiang Goh²

Bertrand Czarny'

Zhi Yang'

Kingshuk Poddar ${ }^{2,3}$

Michal M Dykas 2,3

Abhijeet Patra ${ }^{2,3}$

T Venkatesan ${ }^{2,3}$

Tomasz Panczyk ${ }^{4}$

Chengkuo Lee $^{5}$

Giorgia Pastorin ${ }^{1-3}$

'Department of Pharmacy, National University of Singapore, ${ }^{2} \mathrm{NUS}$ Graduate School for Integrative Sciences and Engineering, Centre for Life Sciences (CeLS), ${ }^{3} \mathrm{NUSNNI}$ NanoCore, National University of Singapore, Singapore; ${ }^{4}$ Institute of Catalysis and Surface Chemistry, Polish Academy of Sciences, Cracow, Poland; ${ }^{5}$ Department of Electrical and Computer Engineering, National University of Singapore, Singapore

Correspondence: Chengkuo Lee Department of Electrical and Computer Engineering, National University of Singapore, 4 Engineering Drive 3,

Singapore 117576

Tel +65 65165865

Email elelc@nus.edu.sg

Giorgia Pastorin

Department of Pharmacy,

National University of Singapore,

Science Drive 2, SI5\#05-PI-03,

Singapore 117543

Tel +6565161876

Fax +65 6779 I554

Email phapg@nus.edu.sg
Abstract: Carbon nanotubes' (CNTs) hollow interior space has been explored for biomedical applications, such as drug repository against undesirable inactivation. To further devise CNTs as smart material for controlled release of cargo molecules, we propose the concept of "gold-carbon nanobottles". After encapsulating cis-diammineplatinum(II) dichloride (cisplatin, CDDP) in CNTs, we covalently attached gold nanoparticles (AuNPs) at the open-tips of CNTs via different cleavable linkages, namely hydrazine, ester, and disulfide-containing linkages. Compared with our previous study in which more than $80 \%$ of CDDP leaked from CNTs in 2 hours, AuNPs were found to significantly decrease such spontaneous release to $<40 \%$. In addition, CDDP release from AuNP-capped CNTs via disulfide linkage was selectively enhanced by twofolds in reducing conditions (namely with $1 \mathrm{mM}$ dithiothreitol [DTT]), which mimic the intracellular environment. We treated human colon adenocarcinoma cells HCT116 with our CDDP-loaded gold-carbon nanobottles and examined the cell viability using lactate dehydrogenase assay. Interestingly, we found that our nanobottles with cleavable disulfide linkage exerted stronger cytotoxic effect in HCT116 compared with normal human fetal lung fibroblast cells IMR-90. Therefore, we infer that our nanobottles strategy with inbuilt disulfide linkage could attain selective release of payload in highly reductive tumor tissues while avoiding collateral damage to normal tissues.

Keywords: carbon nanotubes, surface functionalization, cleavable bonds, cisplatin, drug delivery

\section{Introduction}

Nanosized hollow structures have attracted considerable research interest because of their potential widespread technological applications, such as gas and energy storage, ${ }^{1-3}$ reservoirs to protect biologically active molecules, ${ }^{4}$ tissue engineering, ${ }^{5}$ confined biochemical nanoreactor, ${ }^{6-8}$ separation, ${ }^{9}$ and storage and controlled delivery of chemical and therapeutic molecules. ${ }^{10,11}$ Various nanocontainers (containers with at least one dimension of their inner cavities in nanosized range) made from different nanomaterials, such as polymersomes ${ }^{12}$ and micelles,${ }^{10}$ carbon nanohorns/nanotubes, ${ }^{13,14}$ protein capsids, ${ }^{15}$ and gold nanospheres, ${ }^{16}$ have been reported.

In the realm of drug delivery, a sophisticated nanodelivery system, which is capable of storing and protecting drug molecules, controlling and regulating release of cargo molecules only at target site, is unequivocally desired. In this regard, release of the drugs or active agents from nanocontainers had been successfully demonstrated to be regulated in response to changes in redox potential, ${ }^{17} \mathrm{pH},{ }^{18,19}$ temperature, ${ }^{20}$ light, ${ }^{21}$ and the presence of enzymes found in certain diseases. ${ }^{22}$ The rate of release of the cargo molecules relies on the shape and geometrical properties of nanocontainers. It can also be modulated by nanovalves or nanogates that are responsive to certain environmental changes. ${ }^{19,23}$ 


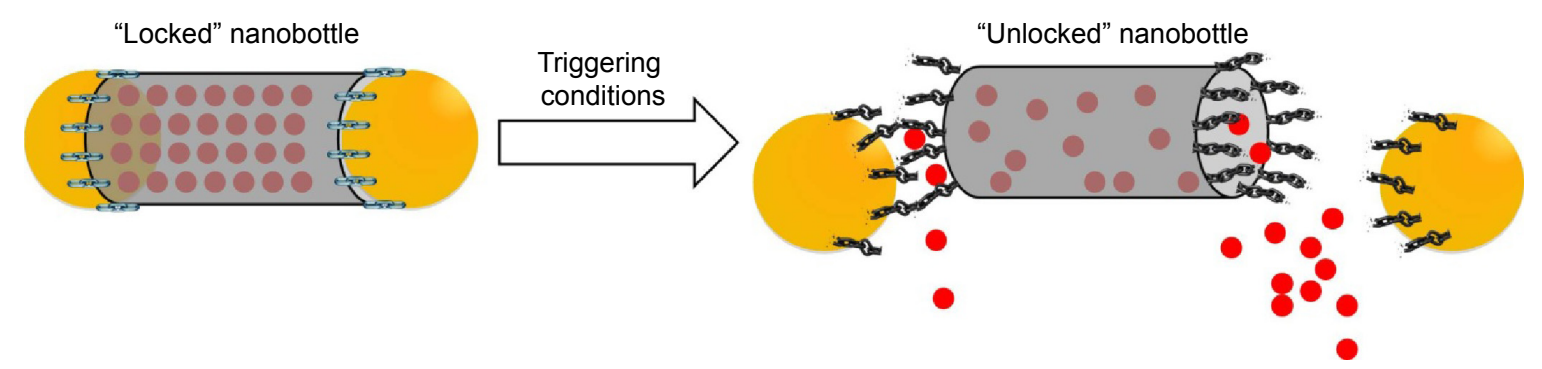

Figure I Design of AuNP-capped carbon nanobottle through cleavable linkers.

Notes: Release of encapsulated content is regulated by the cleavage of the linkers upon specific environmental trigger. Yellow particles are AuNPs (gold nanoparticles). Red particles are the encapsulated CDDP (cisplatin) drug.

Carbon nanotubes (CNTs) have been explored for potential applications in drug delivery, due to favorable attributes such as high aspect ratio, excellent stability, remarkable capability in traversing cellular barriers, and spacious inner cavity for drug loading. ${ }^{24}$ Loading of drugs via entrapment in CNT's hollow space is more desirable as drugs loaded on the outer surface of CNTs risk non-specific activation/ inactivation that stem from premature exposure to the external environment. Compared with the external surface, the interior surface of CNTs possesses more favorable binding energy for adsorption of molecules. ${ }^{25-27}$ Accordingly, the incorporation of guest molecules into the hollow space of CNTs had been demonstrated in various studies. ${ }^{13,28-31}$ However, the open-tips of CNTs are impediment to stable entrapment and controlled release of drug molecules in the hollow space of CNTs. Our group has previously proposed the use of $\mathrm{C}_{60}$ to seal the open-ends of single-walled carbon nanotubes or double-walled carbon nanotubes filled with chemotherapeutics hexamethylmelamine. ${ }^{14}$ However, $\mathrm{C}_{60}$ can only be released using organic solvents such as dichloromethane, and this deters the application of $\mathrm{C}_{60}$-capped carbon nanobottles in the physiological context.

We then developed another capping strategy by using gold nanoparticles (AuNPs) to block the open-tips of multi-walled carbon nanotubes (MWCNTs). ${ }^{32}$ This approach again relied on the physical interaction between interior surface of CNTs and the cap AuNPs. As a result, only AuNPs smaller (ie, $4 \mathrm{~nm}$ ) than the inner diameter of CNTs $(\sim 10 \mathrm{~nm})$ were able to be nano-extracted into the hollow interior cavity and partially narrowed the open-ends of CNTs. This strategy was successful in delaying cis-diammineplatinum(II) dichloride (cisplatin, CDDP) leakage and averting spontaneous release under physiological conditions. Nevertheless, our carbon nanobottles were far from being "smart" in achieving controlled release of cargo molecules only in response to specific trigger.

In this study, we propound another model of carbon nanobottle capped by AuNPs that are covalently appended at the tips of CNTs by cleavable linkers, which are sensitive to external triggers such as low $\mathrm{pH}$, presence of esterase, or reducing thiols (Figure 1). By leveraging on the fact that defect functionalization using strong acid could generate carboxyl groups predominantly localized at the tips of CNTs, ${ }^{33,34}$ we successfully tethered AuNPs (sized similarly as CNTs' inner diameter) at the proximities of CNTs. These AuNPs serve as seal for the open-ends of CNTs and prevent leakage of encapsulated CDDP, a widely used anticancer drug. In addition, we exploited the tumor selectivity of cleavable disulfide linker, which cleaves in response to high reductive environment in tumor cells. We have demonstrated that the capped disulfide carbon nanobottles exhibited stronger growth inhibitory effect on human colon adenocarcinoma cells HCT116 than human fetal lung fibroblast cells IMR-90. Collectively, this cleavable carbon nanobottle could pave the way for devising carbon-based "smart" drug delivery system to attain selective delivery of drugs to tumor cells for enhancing therapeutic outcome.

\section{Experimental}

\section{Materials and instruments}

CDDP, $N$-hydroxysuccinimide (NHS), $N$-(3-dimethylaminopropyl)- $N^{\prime}$-ethylcarbodiimide hydrochloride (EDC $\left.\cdot \mathrm{HCl}\right)$, hydrazine monohydrate, $N, N^{\prime}$-dicyclohexylcarbodiimide (DCC), $N, N$-diisopropylethylamine (DIPEA), 9-mercapto-1nonanol, 3-mercaptopropionic acid (3-MPA), 2-hydroxyethyl disulfide, DL-dithiothreitol (DTT), esterase from porcine liver, ultrapure nitric acid (65\%, for ultratrace analysis), LDH in vitro toxicology assay kit, and McCoy's 5A medium were purchased from Sigma-Aldrich. Nitric acid (65\%) and sulfuric acid (98\%) were purchased from Merck. Spherical AuNPs $(\Phi=40 \mathrm{~nm})$ and conjugated spherical AuNPs $(\Phi=40$ $\mathrm{nm}$, carboxyl polymer) were purchased from Nanopartz ${ }^{\mathrm{TM}}$. The reactions were performed with solvents purchased from Tedia. Spectra Por dialysis membrane $(12,000-14,000 \mathrm{Da}$ molecular weight cutoff) was used in the drug release 
experiment. Omnipore hydrophilic polytetrafluoroethylene (PTFE) membranes $(0.2 \mu \mathrm{m})$ and fluoropore hydrophobic PTFE membranes $(0.22 \mu \mathrm{m})$ were purchased from EMD Millipore (Billerica, MA, USA). Ultrapure MWCNT was kindly provided by Prof Ramaprabhu (Nano-Functional Materials Technology Centre [NFMTC], Indian Institute of Technology, Chennai, India): the outer and inner diameters of ultrapure MWCNT (purity $>95 \%$ ) are $30-40 \mathrm{~nm}$ and approximately $10 \mathrm{~nm}$, respectively; their length ranged from a few hundred nanometers to several microns. Hyclone ${ }^{\mathrm{TM}}$ fetal bovine serum (FBS), TrypleExpress ${ }^{\mathrm{TM}}$ dissociating agent, and Dulbecco's Modified Eagle's Medium (DMEM) were procured from Thermo Fisher Scientific Inc. (Waltham, MA, USA).

Transmission electron microscopy (TEM) was carried out using JEM 2010F HR-TEM (JEOL, Tokyo, Japan). UV-vis spectra were recorded on a BioSpec-nano (SHIMADZU, Kyoto, Japan). Thermogravimetric analysis (TGA) using TGA-SDT 2960 Simultaneous DTA-TGA and ICP-OES using Perkin Elmer Dual-view Optima 5300 DV Inductively Coupled Plasma Optical Emission Spectrometer were performed by the Chemical, Molecular and Materials Analysis Centre (National University of Singapore, Singapore).

\section{Functionalization of MWCNTs Oxidation of MWCNTs}

Ultrapure pristine MWCNTs (10 mg) were oxidized by ultrasonication in the presence of acidic mixture composed of $95 \%$ sulfuric acid and $65 \%$ nitric acid $(3: 1 \mathrm{v} / \mathrm{v})$ for 6 hours. The MWCNTs/acid mixture was diluted with deionized water and filtered through $0.2 \mu \mathrm{m}$ hydrophilic PTFE membrane. The residue was washed extensively with deionized water until the $\mathrm{pH}$ turned to neutral. The resulting MWCNTs were dried in vacuo to yield oxidized MWCNTs (MWCNT-OX).

\section{Synthesis of $f-M W C N T-I$}

MWCNT-OX (5.4 mg) was suspended in thionyl chloride $(6 \mathrm{~mL})$ and refluxed for 24 hours. The resulting solid $(1.6 \mathrm{mg})$ was dried in vacuo and re-dispersed in dry tetrahydrofuran (THF, $0.4 \mathrm{~mL}$ ), followed by dropwise addition of mixture of hydrazine monohydrate $(2.1 \mathrm{mmol})$ and triethylamine $(10 \mathrm{mmol})$. The resulting mixture was stirred at room temperature for 24 hours and filtered through a hydrophilic PTFE membrane. The residue was washed with deionized water and dried in vacuo to yield $f$-MWCNT-1.

\section{Synthesis of $f$-MWCNT-2}

MWCNT-OX (4.0 mg) was suspended in THF (1 mL) under sonication. NHS (28 $\mu \mathrm{mol})$, DCC (92 $\mu \mathrm{mol})$, and 2-hydroxyethyl disulfide (92 $\mu \mathrm{mol})$ were added and the mixture was stirred at room temperature for 24 hours. The MWCNTs were filtered through a hydrophilic PTFE membrane and washed with DMF and deionized water. The remaining solid was dried in vacuo to yield $f$-MWCNT-2.

\section{Functionalization of AuNPs}

Reaction with 4-(2-(2-(2-mercaptoethoxy)ethoxy) ethoxy)benzaldehyde

The reaction between 4-(2-(2-(2-mercaptoethoxy)ethoxy) ethoxy)benzaldehyde and AuNPs was carried out as reported in our previous work. ${ }^{32}$ Briefly, a solution of compound 1 $(0.7 \mathrm{mg})$ in THF was added to spherical AuNPs $(0.37 \mathrm{mg})$ colloidal suspension under sonication. The mixture was agitated for 24 hours and subjected to four cycles of washing to remove unbound 4-(2-(2-(2-mercaptoethoxy)ethoxy) ethoxy)benzaldehyde. The modified AuNPs were suspended in THF to yield the colloidal suspension of $f$-AuNP-1.

\section{Reaction with 9-mercapto-I-nonanol}

A solution of 9-mercapto-1-nonanol $(0.28 \mathrm{mg})$ in $50 \%$ ethyl alcohol (EtOH) was added to spherical AuNPs $(0.28 \mathrm{mg})$ colloidal suspension under sonication. The mixture was agitated for 20 hours and subjected to four cycles of washing to remove unbound 9-mercapto-1-nonanol. The modified AuNPs were suspended in THF to yield the colloidal suspension of $f$-AuNP-2.

\section{Preparation of AuNP-capped nanobottles via cleavable linkers}

AuNP-capped nanobottles via hydrazone linkage

We entrapped CDDP within $f$-MWCNT-1 by nanoextraction, ${ }^{32}$ yielding $f$-MWCNT-1-CDDP. The open-ends of $f$-MWCNT-1-CDDP were capped by mixing the CNTs $(4.2 \mathrm{mg})$ with $f$-AuNP-1 $(0.37 \mathrm{mg})$ in THF $(1 \mathrm{~mL})$ under sonication, followed by addition of $0.01 \mathrm{M} \mathrm{HCl}(20 \mu \mathrm{L})$. The resulting mixture was stirred at room temperature for 4 days. The resulting product was filtered through a PTFE membrane and washed with THF, EtOH, and deionized water. The remaining solid was dried in vacuo to yield the capped CDDP@MWCNT nanobottles assembled via hydrazone bond (CDDP@f-MWCNT-1).

\section{AuNP-capped nanobottles via ester linkage}

Encapsulation of CDDP into MWCNT-OX was accomplished also by using nano-extraction, yielding CDDP@ MWCNT-OX. As-prepared f-AuNP-2 (0.28 mg), DCC ( $7.8 \mu \mathrm{mol})$, NHS $(4.7 \mu \mathrm{mol})$, and catalytic amount of DIPEA were added to the suspension of CDDP@MWCNT-OX in 
THF ( $1 \mathrm{~mL})$. The mixture was stirred at room temperature for 2 days and filtered through a PTFE membrane. The residue was washed with THF and EtOH, and was dried in vacuo to yield the capped CDDP@MWCNT nanobottles assembled via ester bond (CDDP@f-MWCNT-2).

\section{AuNP-capped nanobottles via disulfide linkage}

We entrapped CDDP within $f$-MWCNT-2 by nano-extraction, yielding $f$-MWCNT-2-CDDP. $f$-MWCNT-2-CDDP (2.8 mg) and carboxyl conjugated AuNPs $(1.5 \mathrm{mg})$ were then sonicated in THF ( $1 \mathrm{~mL})$. DCC (17.3 $\mu \mathrm{mol})$, NHS $(10.4 \mu \mathrm{mol})$, and DIPEA were later added into the suspension. The mixture was stirred at room temperature for 5 days. The resulting product was filtered through a PTFE membrane and washed with THF, EtOH, and deionized water. The remaining solid was dried in vacuo to yield the capped CDDP@MWCNT nanobottles assembled via disulfide bond (CDDP@fMWCNT-3).

\section{In vitro release of CDDP from covalently capped CDDP@MWCNTs nanobottles}

In vitro release of CDDP from covalently capped CDDP@ MWCNT nanobottles was evaluated using a dialysis bag diffusion method as previously reported. ${ }^{32}$ Briefly, capped CDDP@MWCNTs were dispersed in deionized water $(200 \mu \mathrm{L})$, transferred to a dialysis button with 12,000-14,000 Da cutoff and immersed in release medium (400 mL).CDDP@f-MWCNT-1, which contains pH-sensitive hydrazone bond, was expected to unleash its content at low $\mathrm{pH}$. Therefore, the in vitro release was carried out in media at two different pHs, ie, Tris buffer ( $\mathrm{pH}$ 7.2) or MES buffer ( $\mathrm{pH} 5.5$ ). On the other hand, to investigate the release of CDDP from CDDP@f-MWCNT-2, the samples were immersed in two different release media, namely phosphate-buffered saline (PBS) containing $5 \mathrm{U} / \mathrm{mL}$ esterase from porcine liver or PBS without esterase. Meanwhile, for CDDP@f-MWCNT-3, in vitro release was performed in PBS containing $1 \mathrm{mM}$ DTT or pristine PBS. An aliquot $(2 \mathrm{~mL})$ of sample was collected from the release medium after 2 and 72 hours, and the platinum levels were determined using ICP-OES.

\section{Cell culture}

Human colon adenocarcinoma cells (HCT116) were chosen as a cancer cell prototype with overexpressed glutathione (GSH) for cytotoxicity assay for CDDP@f-MWCNT-3 nanobottles containing disulfide linkage. Human fetal lung fibroblast cells (IMR-90) were chosen as a non-cancerous control to investigate the selectivity of CDDP@f-MWCNT-3 nanobottles. HCT116 was obtained courtesy of Prof Victor Yu (National University of Singapore, Singapore) and human fetal lung IMR-90 was purchased from American Type Culture Collection (ATCC, Manassas, VA, USA).

HCT116 cells were cultured in McCoy's 5A supplemented with $10 \%$ FBS while IMR-90 cells were cultured in DMEM supplemented with 10\% FBS. The cells were allowed to grow at $37^{\circ} \mathrm{C}$ in humidified air containing $5 \% \mathrm{CO}_{2}$. All cells were subcultured every $3-4$ days using TrypleExpress ${ }^{\mathrm{TM}}$ dissociating agent.

All the experiments were performed on cells that were either purchased or kindly provided by collaborators. Where possible, we tried to minimize the amount of cells used, as well as the eventual risks associated with the handling of these cell lines. All the people who performed the studies underwent vaccination and our laboratory has been certified by the Office of Safety, Health \& Environment (OSHE) at the National university of Singapore to perform these experiments.

\section{Cytotoxicity assessment by lactate dehydrogenase (LDH) assay}

The efficacy of various CDDP-based drug-constructs on HCT116 cells and IMR-90 cells was studied using lactate dehydrogenase (LDH) assay. The corresponding concentrations of MWCNTs were back-calculated from \% loading determined by ICP-OES analysis, ie, 40.1\% w/w and 59.5\% w/w for CDDP@f-MWCNT-3 and CDDP@MWCNT-OX, respectively. Table $\mathrm{S} 1$ summarizes the concentrations of CDDP,CDDP@f-MWCNT-3,CDDP@MWCNT-OX, and blank nanobottles employed in this study.

The cultured cells were seeded at a density of 5,000 cells per well in 96-well plates and allowed to adhere for 24 hours prior to drug treatment. The cells were then treated with $100 \mu \mathrm{L}$ of medium containing different concentrations $(0.1-30 \mu \mathrm{M})$ of CDDP@f-MWCNT-3, CDDP@MWCNT-OX, or blank nanobottles for 6 hours. Later on, replacement with fresh media without CDDP or CDDP-CNT constructs was performed and the cells were allowed to grow for another 66 hours.

A modified version of LDH assay was used to access cell viability to avoid any interference from CNTs. ${ }^{35}$ The assay measures LDH content in intact cells that survived the treatment, instead of the $\mathrm{LDH}$ released into the media upon treatment-induced cell death. Briefly, cells were lysed with $10 \mu \mathrm{L}$ of lysis buffer $(0.9 \%$ Triton X-100) mixed with $100 \mu \mathrm{L}$ FBS supplemented culture media (phenol-red free) 
for 60 minutes at $37^{\circ} \mathrm{C}$. As CNTs interfere with absorbance, they were pelleted down by centrifugation at $13,000 \times g$ for 5 minutes. According to manufacturer's instructions, $50 \mu \mathrm{L}$ of the supernatant of the centrifuged cell lysate was mixed with $50 \mu \mathrm{L}$ of LDH substrate mixture (prepared from LDH in vitro toxicology assay kit) and the mixture was allowed to incubate at room temperature for 30 minutes. The absorbance at $490 \mathrm{~nm}$ was obtained by TECAN infinite M200 PRO. The amount of LDH detected represented the number of cells that survived the treatment. The percentage of cell survival was calculated using the following equation:

$$
\% \text { cell survival }=\frac{A_{490 \mathrm{~nm}} \text { of treated cells }}{A_{490 \mathrm{~nm}} \text { of untreated cells }}
$$

where $A_{490 \mathrm{~nm}}$ is the absorbance at $490 \mathrm{~nm}$.

\section{Statistical analysis}

All statistical analyses were accomplished using Statistical Package for the Social Sciences (SPSS, Chicago, IL, USA). Data are presented as mean \pm standard deviation. Statistical significance was calculated using Student's $t$-test.
Differences with $P<0.05$ were considered as statistically significant.

\section{Results and discussion Synthesis of AuNP-capped MWCNT nanobottles filled with CDDP}

To generate carbon nanobottles that could achieve controlled release of incorporated drugs, we proposed the capping strategy by covalently tethering AuNPs via cleavable linkages that are sensitive to pathological manifestations of cancer such as low $\mathrm{pH}$, presence of abundant esterase, and thiols. ${ }^{36}$ In accord, three linkers consisting of different cleavable bonds, namely hydrazone-, ester-, and disulfide-bond, were employed to append AuNPs at MWCNT open-ends. The capping agent AuNPs of $40 \mathrm{~nm}$ in diameter were selected to match MWCNT's inner diameter (ranging from 30 to $40 \mathrm{~nm}$ ). Bare AuNPs were modified with either 4-(2-(2-(2-mercaptoethoxy) ethoxy)ethoxy)benzaldehyde or 9-mercapto-1-nonanol to generate functional groups essential for forming the cleavable hydrazone or ester containing linkers, respectively (Figure 2). Meanwhile, commercially available carboxylated AuNPs were directly used as a sealing agent for MWCNTs via disulfide linkage.
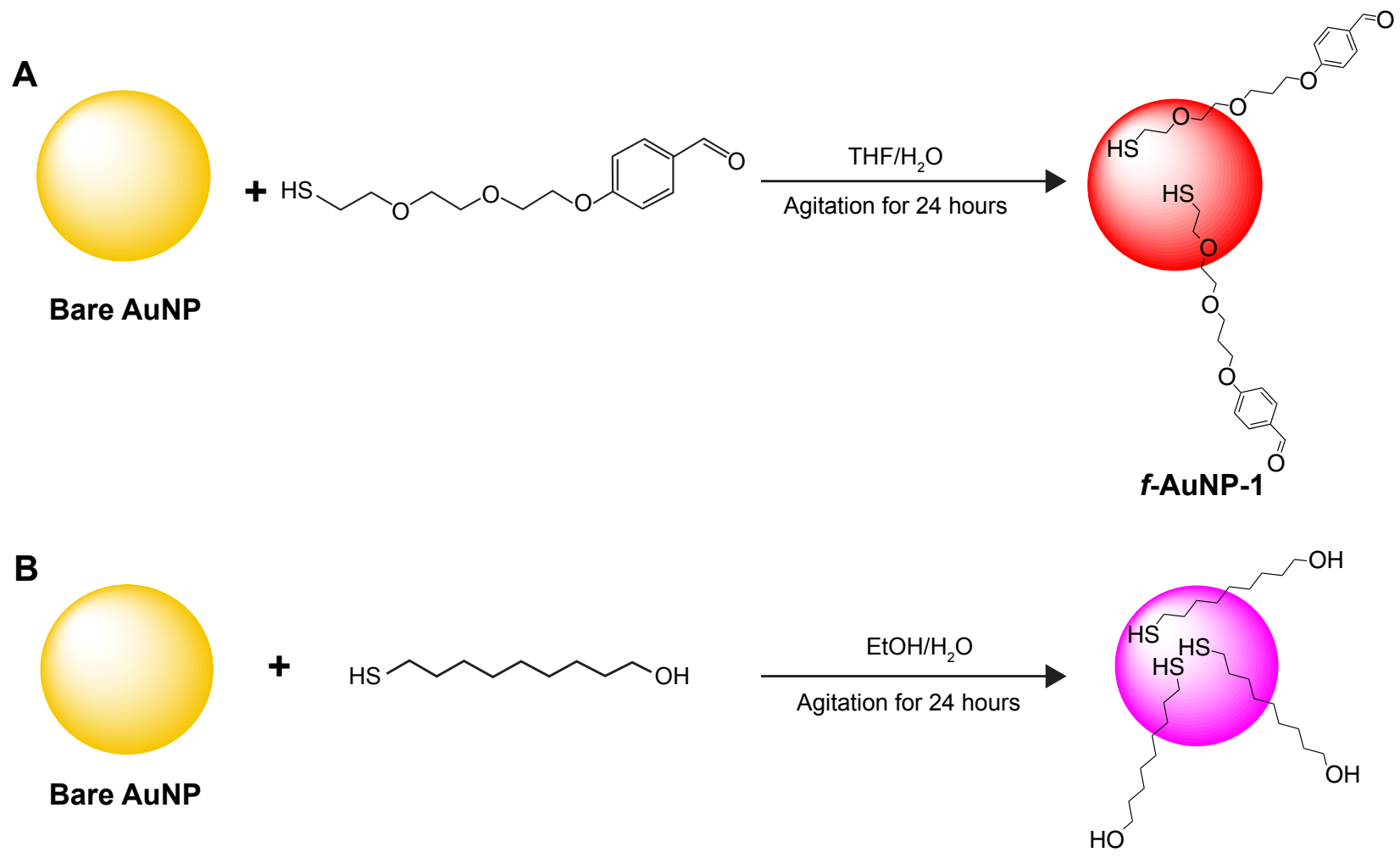

f-AuNP-2

Figure 2 Functionalization of AuNPs with (A) 4-(2-(2-(2-mercaptoethoxy)ethoxy)ethoxy)benzaldehyde and (B) 9-mercapto-I-nonanol. Abbreviations: AuNPs, gold nanoparticles; EtOH, ethyl alcohol; THF, tetrahydrofuran. 
Functionalization of 4-(2-(2-(2-mercaptoethoxy)ethoxy) ethoxy)benzaldehyde on AuNPs was qualitatively validated by high-resolution imaging using TEM. The high electron density of AuNPs renders a high contrast under TEM (Figure 3A), while a layer of shell with lower contrast was observed on $f$-AuNP-1 (Figure 3B). This indicates the presence of 4-(2-(2-(2-mercaptoethoxy)ethoxy)ethoxy) benzaldehyde, which is lower in electron density compared with AuNPs. As for $f$-AuNP-2, we also obtained similar observation (Figure S1). The functionalization of linker molecules on AuNPs was confirmed by UV-VIS spectroscopy. Upon functionalization with 4-(2-(2-(2-mercaptoethoxy)ethoxy) ethoxy)benzaldehyde, the absorbance of AuNPs was shifted from 527 to $536 \mathrm{~nm}$ (Figure S2).

On the other hand, MWCNTs were modified via defect functionalization in order to generate reactive groups (mainly at the tips $)^{33}$ for appendage of $f$-AuNPs (Figure 4). Briefly, pristine MWCNTs were oxidized (MWCNT-OX) with strong acid to generate reactive carboxyl groups. We verified the integrity of MWCNT-OX by visualizing them using TEM and a significant reduction in length was observed after oxidation (Figure S3). To obtain AuNP-capped MWCNTs via hydrazone linkage, carboxylic acids on MWCNT-OX were activated by acyl chlorides and reacted with hydrazine monohydrate to generate hydrazide groups ( $f$-MWCNT-1) (Figure 4). Meanwhile, in order to introduce disulfide linkage, MWCNTs were functionalized with 2-hydroxyethyl disulfide (f-MWCNT-2) (Figure 4).
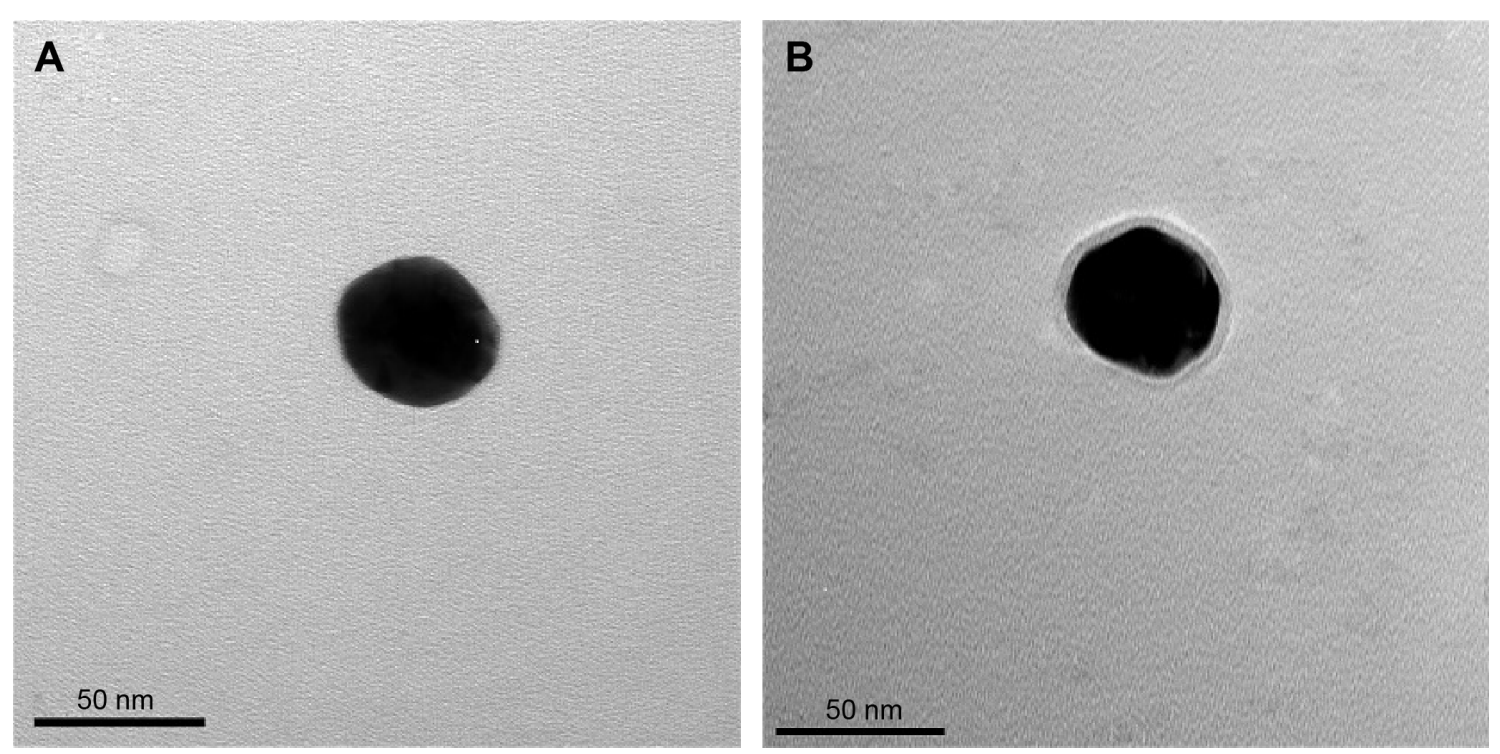

Figure 3 TEM images of (A) bare AuNPs and (B) f-AuNP-I. A layer of shell with less contrast than AuNPs was observed after functionalization with 4-(2-(2-(2-mercaptoethoxy) ethoxy)ethoxy)benzaldehyde.

Abbreviations: AuNPs, gold nanoparticles; TEM, transmission electron microscopy.

CDDP was encapsulated in $f$-MWCNT-1, MWCNT-OX, or $f$-MWCNT-2 by nano-extraction. ${ }^{32}$ The open-ends of MWCNTs were capped by covalently appending AuNPs via cleavable linker (containing hydrazone, ester, or disulfide bond).In accord, the hydrazide groups on CDDP containing $f$-MWCNT-1 were reacted with benzaldehyde groups on $f$-AuNP-1, forming AuNP-capped $f$-MWCNT-1 nanobottles via hydrazone linkage that is sensitive to acidic $\mathrm{pH}$ (CDDP@f-MWCNT-1) (Figure 5A). Meanwhile, the carboxylic acid groups on MWCNT-OX were directly conjugated with $f$-AuNP-2 (CDDP@f-MWCNT-2) via ester linkage using carbodiimide-mediated coupling (Figure 5B). To cap CDDP encapsulated $f$-MWCNT-2 containing disulfide functionalities, the terminal hydroxyl group on MWCNTs was conjugated with carboxylic acids of carboxyl AuNPs via carbodiimidemediated coupling (CDDP@f-MWCNT-3) (Figure 5C).

The CDDP containing MWCNTs capped by AuNPs via different linkers was visualized at high resolution using TEM. AuNPs were observed at the open-tips of $f$-MWCNT-1 as well as sidewall defects of most of nanotubes (Figure S4). A zoomed image of CDDP@f-MWCNT-2 and CDDP@f-MWCNT-3 samples (Figures 6 and S5) clearly showed AuNPs sealing the tips of the tubes in the form of a "nanobottle", although a minority of tips were still left open. Our findings resonate with the observations reported by Zhao et al, whereby AuNPs with commensurate diameters to carbon nanotube cups successfully corked the open rim of the cup. ${ }^{37}$ In addition, it is reported in the same study that 


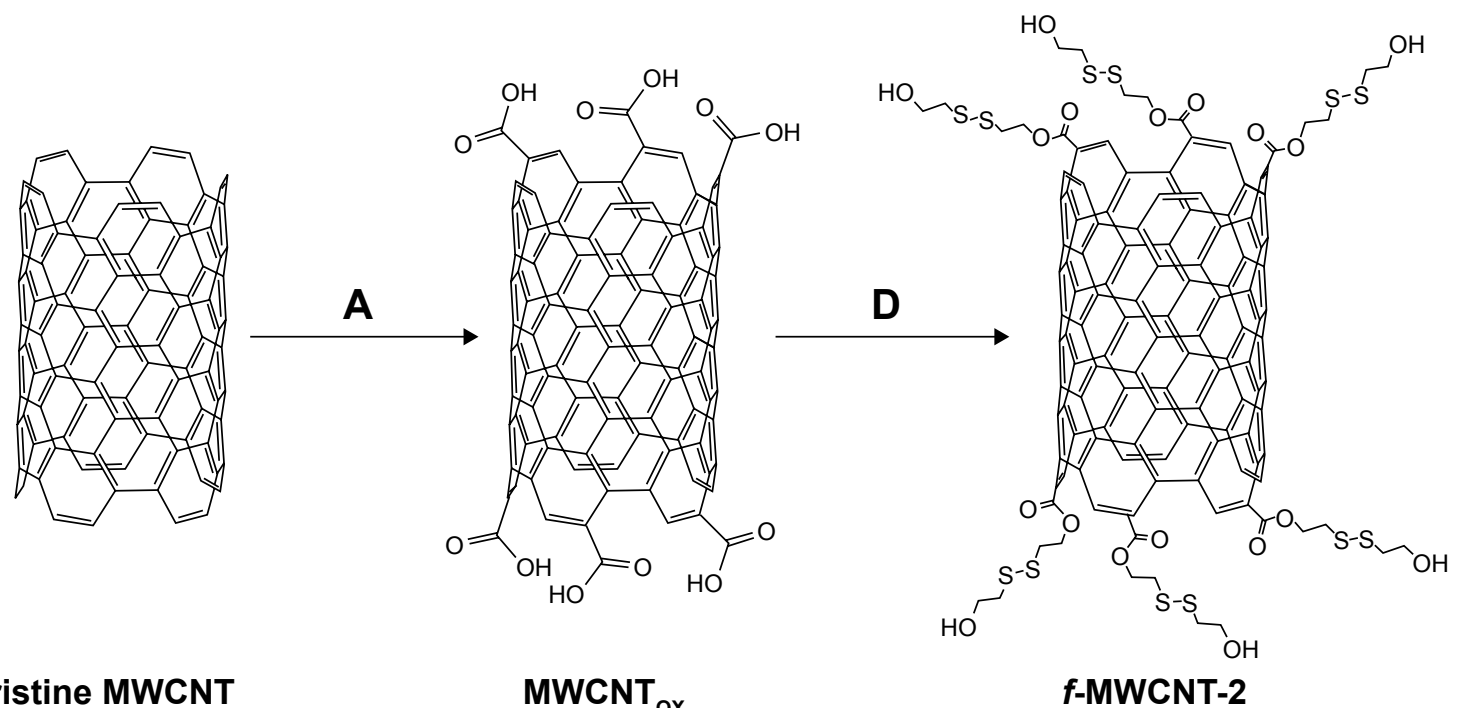

Pristine MWCNT

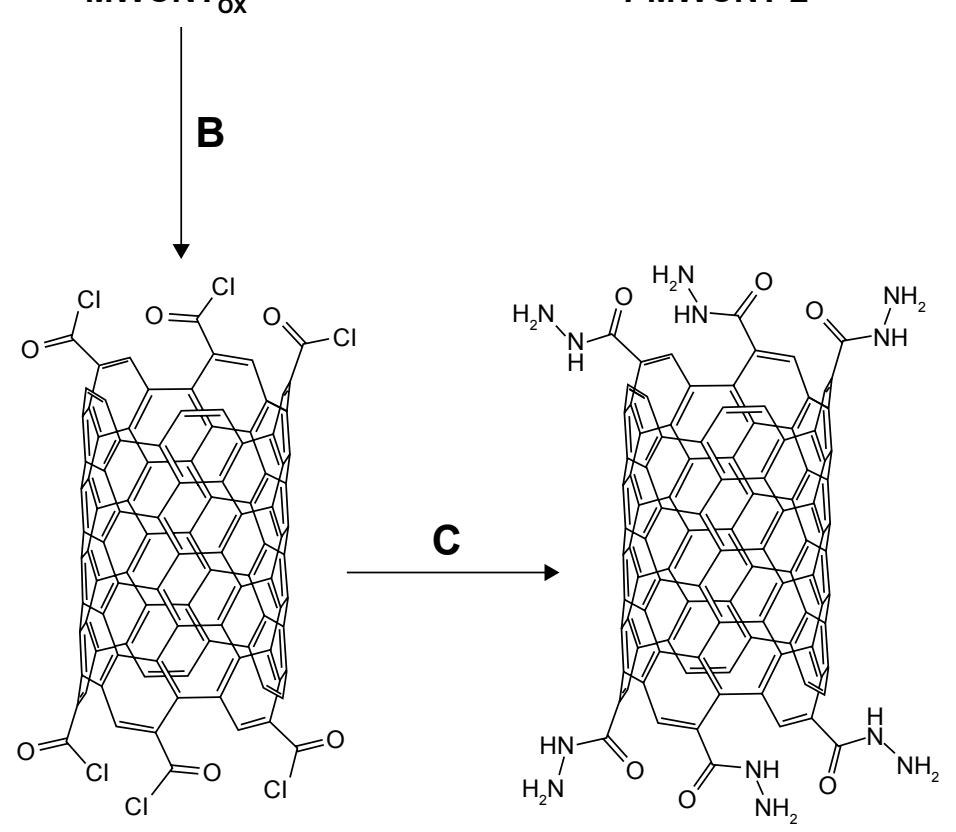

f-MWCNT-1

Figure 4 Functionalization of MWCNTs.

Notes: (A) $\mathrm{H}_{2} \mathrm{SO}_{4} / \mathrm{HNO}_{3}(3: \mathrm{l} / \mathrm{v})$, sonication for 6 hours; (B) SOCl , reflux for 24 hours; (C) hydrazine monohydrate, THF, TEA for 24 hours; and (D) 2 -hydroxyethyl disulfide, DCC, NHS, THF for 24 hours.

Abbreviations: AuNPs, gold nanoparticles; DCC, N,N'-dicyclohexylcarbodiimide; MWCNTs, multi-walled carbon nanotubes; TEA, triethylamine; NHS, N-hydroxysuccinimide; THF, tetrahydrofuran.

generation of localized reactive sites with amine functionalities was possible using nitrogen doping of CNTs during chemical vapor deposition synthesis. As a result, AuNPs were mainly found to be distributed at the open rim of the cup. In short, our findings support the hypothesis that AuNPs with size comparable to MWCNTs diameter could effectively block the open-tips of MWCNTs and this prompted us to profile the release of CDDP from capped MWCNTs. Moreover, while it is true that CNTs' length might affect the encapsulation of cisplatin and the subsequent release profile, this aspect was not applicable to our samples, which were all oxidized by ultrasonication in the presence of acidic mixture composed of $95 \%$ sulfuric acid and $65 \%$ nitric acid $(3: 1 \mathrm{v} / \mathrm{v})$ for 6 hours. These conditions were previously reported to shorten our tubes' length from a few microns to a range of approximately $200-900 \mathrm{~nm}^{38}$ (Figure S3). All the samples had the same external diameter $(38 \mathrm{~nm}$ in average, $n=144, \sigma=13.6$ ) and aspect ratio in the range of $19-21,{ }^{39}$ which therefore had the same influence in terms of release profiles. 
A

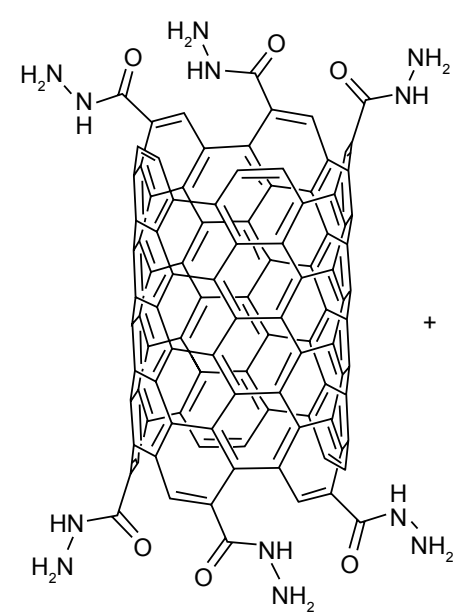

f-MWCNT-1

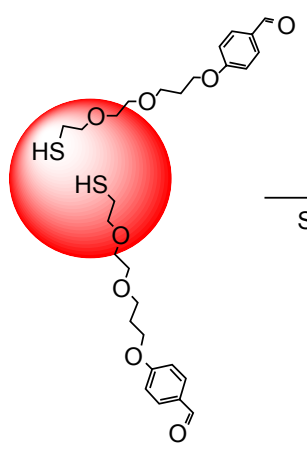

f-AuNP-1
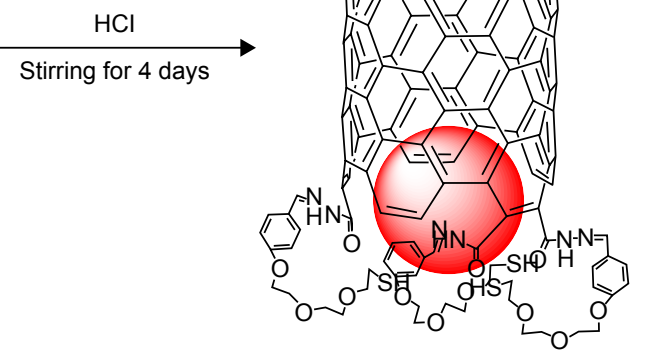

CDDP@f-MWCNT-1

B
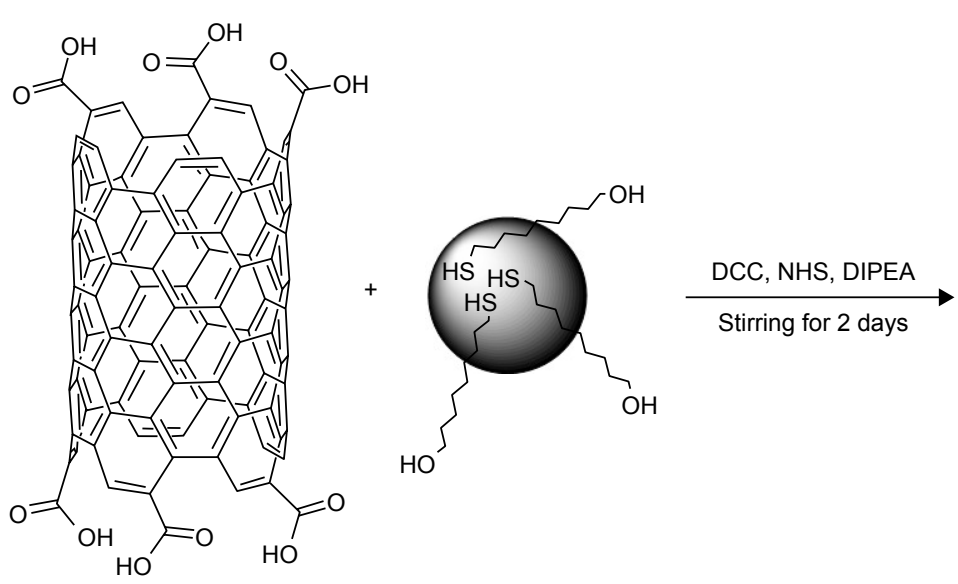

MWCNT $_{\text {ox }}$

f-AuNP-2

C

HO

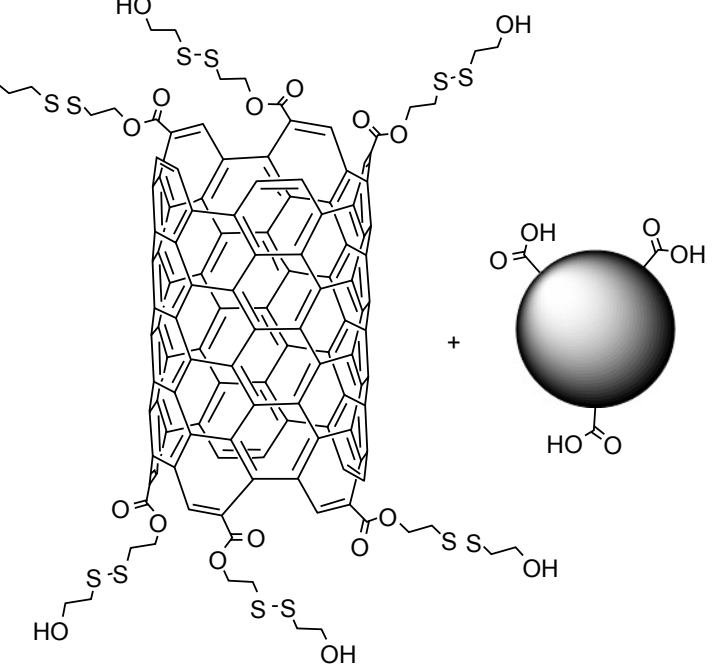

$\underset{\text { Stirring for } 5 \text { days }}{\stackrel{\text { DCC, NHS, DIPEA }}{\longrightarrow}}$

$f$-MWCNT-2

Carboxyl AuNP

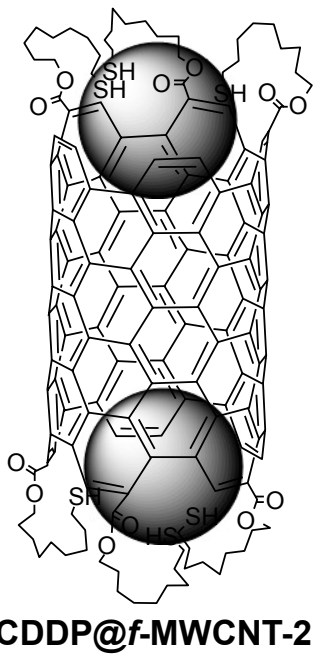

CDDP@f-MWCNT-2

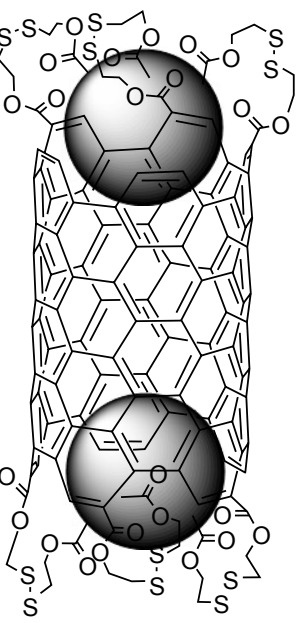

CDDP@f-MWCNT-3

Figure 5 Preparation of AuNP-capped CDDP@MWCNT nanobottles.

Notes: (A) CDDP@f-MWCNT-I containing hydrazone linkage; (B) CDDP@f-MWCNT-2 containing ester linkage; and (C) CDDP@f-MWCNT-3 containing disulfide linkage. Abbreviations: AuNPs, gold nanoparticles; CDDP, cis-diammineplatinum(II) dichloride; DCC, N,N'-dicyclohexylcarbodiimide; DIPEA, N,N-diisopropylethylamine; MWCNT, multi-walled carbon nanotube; NHS, N-hydroxysuccinimide. 

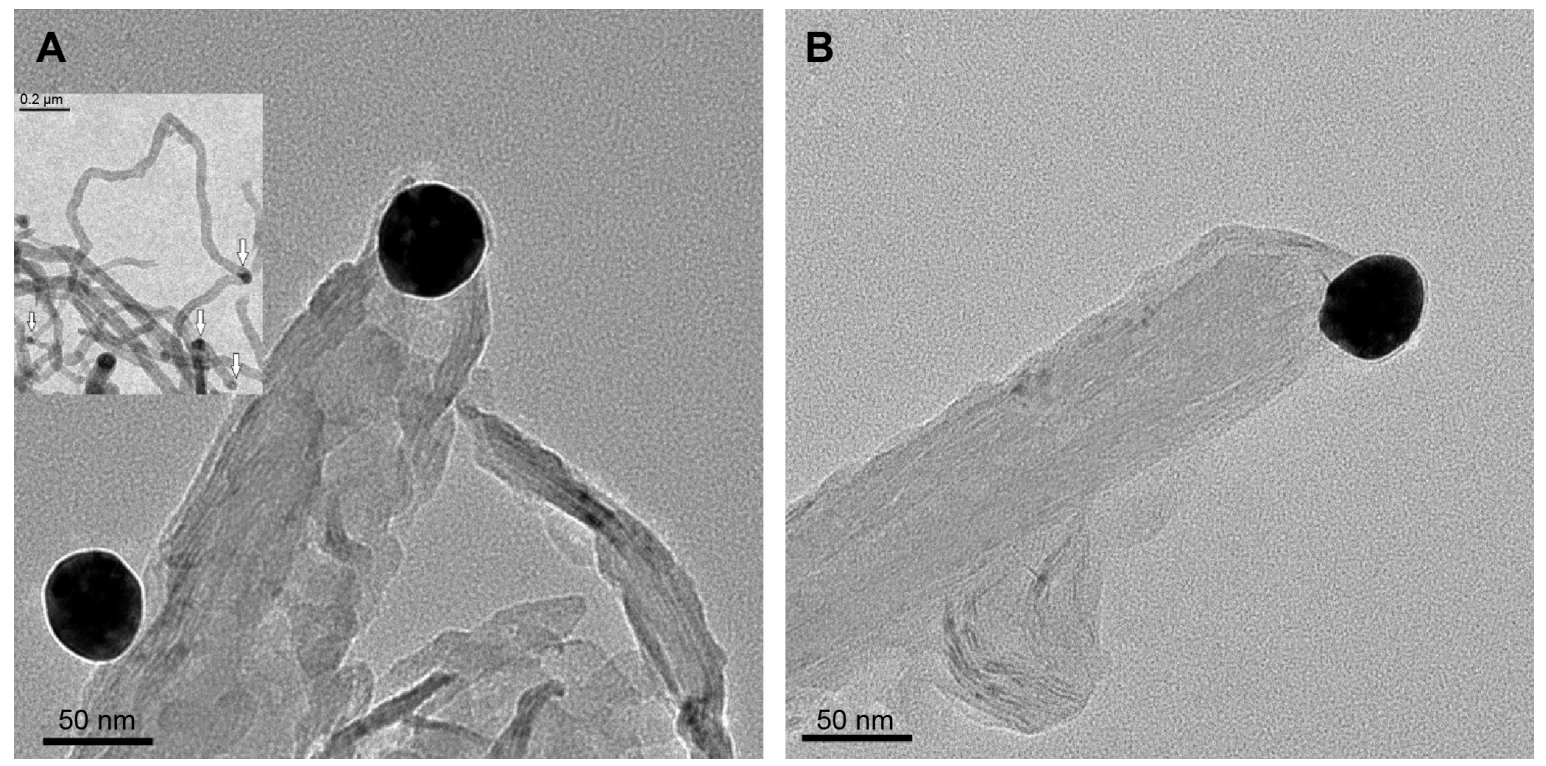

Figure 6 TEM images of (A) CDDP@f-MWCNT-2 composed of MWCNT, AuNP, and ester linkage and (B) CDDP@f-MWCNT-3 composed of MWCNT, AuNP, and disulfide linkage. Inset in (A): HRTEM of CDDP@f-MWCNT-2 showing the walls of MWCNTs and suggesting the presence of tubes with both ends capped with gold nanoparticles (white arrows).

Abbreviations: AuNPs, gold nanoparticles; CDDP, cis-diammineplatinum(II) dichloride; HRTEM, high-resolution TEM; MWCNT, multi-walled carbon nanotube; TEM, transmission electron microscopy.

\section{In vitro release of AuNP-capped nanobottles}

We examined the in vitro release of CDDP from CDDP@fMWCNT-1, CDDP@f-MWCNT-2, and CDDP@fMWCNT-3, respectively, at different simulative conditions to facilitate the cleavage of AuNPs from MWCNTs (Figure 7). AuNPs were appended at the open-tips of $f$-MWCNT-1 via hydrazone linkage, which is sensitive to acidic $\mathrm{pH} .{ }^{40}$

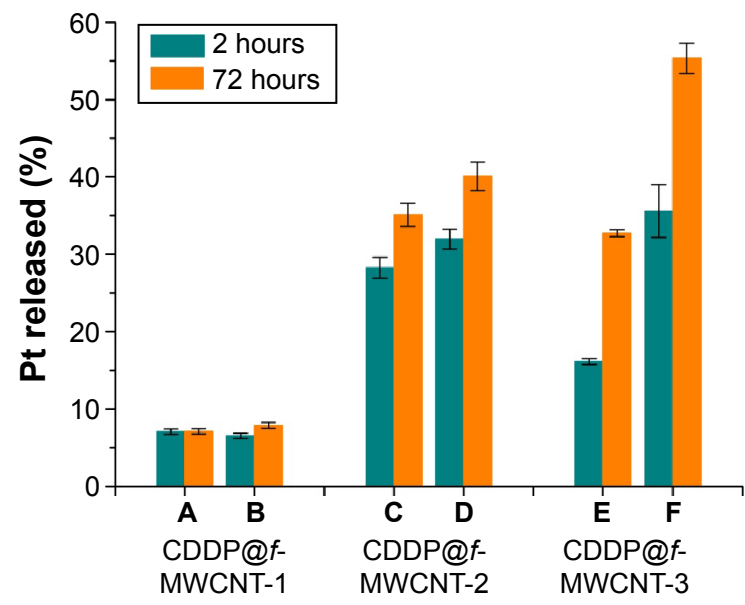

Figure 7 Release of Pt from covalently capped nanobottles of CDDP@f-MWCNT-1, CDDP@f-MWCNT-2, and CDDP@f-MWCNT-3 under various conditions.

Notes: (A) Tris buffer at pH 7.2; (B) MES buffer at pH 5.5; (C) PBS; (D) PBS containing $5 \mathrm{U} / \mathrm{mL}$ esterase from porcine liver; (E): PBS; and (F) PBS containing I mM DTT. Abbreviations: CDDP, cis-diammineplatinum(II) dichloride; DTT, dithiothreitol; MWCNT, multi-walled carbon nanotube; PBS, phosphate-buffered saline; MES, 2-(N-morpholino)ethanesulfonic acid.
Hence, we postulated that this delivery system could effectively release its payload at tumor microenvironment or in endolysosomal compartment that is acidic in nature. $f$-MWCNT-2 contains ester linkage, which has also been commonly used to facilitate controlled release of drug molecules from nanocarriers or prodrugs upon hydrolysis. ${ }^{41}$ On the other hand, disulfide bond containing linkers were also included to attach AuNPs on MWCNTs open-tips. Disulfide linkage is widely known to be sensitive to reducing agents such as DTT or intracellular GSH. Hence, the disulfide linkage could facilitate controlled release of loaded cargo intracellularly where GSH level is significantly higher $(0.5-10 \mathrm{mM})$ than extracellular environment $(2-20 \mu \mathrm{M}){ }^{42}$ Moreover, as GSH levels have been consistently reported to be much higher (up to 1,000 times) in tumor cells compared with adjacent non-malignant tissue,${ }^{43-45}$ our strategy is conceived to confer selectivity against cancerous cells.

The CDDP release from CDDP@f-MWCNT-1 nanobottles was performed at $\mathrm{pH}$ 5.5. Unexpectedly, CDDP release from $f$-MWCNT-1 was found to be limited at both acidic and control neutral pHs (Figure 7A and B). Although leakage of CDDP from MWCNTs ${ }^{32}$ has been resolved with the use of covalently attached AuNPs seal, the hydrazone linkage employed in CDDP@f-MWCNT-1 was ostensibly insusceptible to acidic condition, hence precluding the desired controlled release of CDDP. On the other hand, in vitro release of CDDP from CDDP@f-MWCNT-2 was 
conducted by presenting esterases in release medium. AuNPs capped on MWCNTs through ester linkage were able to reduce the leakage of CDDP to a lesser extent than CDDP@fMWCNT-1. Nevertheless, the presence of triggering esterase again did not result in enhanced CDDP release, suggesting that AuNPs were not efficiently removed with $5 \mathrm{U} / \mathrm{mL}$ esterase (Figure 7C and D). We reasoned that inherited steric hindrance existed between AuNPs and MWCNTs, which might have impacted access of triggering agents in reaching cleavable linkage, thus compromising the desired controlled release under designated conditions.

As regards CDDP@f-MWCNT-3 nanobottles containing both ester bond and disulfide bond, the platinum content detected in the release medium was remarkably higher in the presence of $1 \mathrm{mM}$ DTT compared with PBS control (Figure 7E and F), suggesting that DTT played a part in the removal of caps and enabled the release of encapsulated CDDP, although the concentration of disulfide bond nanobottles in release medium was comparable to hydrazone bond and ester bond nanobottles. The success in caps removal was presumably attributed to the higher sensitivity of disulfide bond in nanobottles to DTT, as well as the kinetics of thiol-disulfide exchange reactions, in which a thiolate group attacks a sulfur atom of a disulfide bond to form a new disulfide bond and the other sulfur atom of the original disulfide bond is released as a new thiolate. ${ }^{46,47}$ These results are in agreement with the work reported by Chen et al, whereby the fluorescein-filled CNTs, whose ends were plugged by the addition of thiol-silica nanospheres via the formation of a disulfide linkage, displayed a selective discharge process triggered by exposure to DTT or at elevated temperature; moreover, the release rate of encapsulated fluorescein could be controlled through increased DTT concentration or temperature, respectively. ${ }^{48}$ On the other hand, it should be noted that some encapsulated CDDP was also found to leak from CDDP@f-MWCNT-3 nanobottles in PBS, as observed in CDDP@f-MWCNT-1 and CDDP@f-MWCNT-2 nanobottles, due to the incomplete capping of nanotubes. Future work will attempt to enhance the sealing efficiency and to provide higher resolution images. Overall, the CDDP@f-MWCNT-3 nanobottles represent a better choice for controlled release in comparison with the other two types of nanobottles.

\section{Cytotoxicity of CDDP, CDDP@f- MWCNT-3, CDDP@MWCNT-OX, in HCTII 6 and IMR-90}

Based on the findings of in vitro release study, we selected only CDDP@f-MWCNT-3 for subsequent cytotoxicity examination as the other constructs CDDP@f-MWCNT-1 and CDDP@f-MWCNT-2 exhibited poor controlled release of CDDP. HCT116 colon carcinoma was selected as cancer cell prototypes that overexpress $\mathrm{GSH} .{ }^{49}$ Cells were incubated for 6 hours with CDDP-containing samples (or controls) in order to mimic the clinically used intravenous (iv) infusion of cisplatin, and then further incubated for 66 hours in fresh media to assess the cytotoxicity profiles over a period of 72 hours.

Addition of free CDDP to HCT116 cells induced dose-dependent cytotoxicity with half maximal inhibitory concentration $\left(\mathrm{IC}_{50}\right)$ falling within the range of $4-8 \mu \mathrm{M}$ (Figure 8A). In contrast, CDDP treatment to normal lung fibroblast IMR-90 was inefficient in impairing cell viability, highlighting substantial selectivity of CDDP treatment in curbing cancer cell growth. CDDP@f-MWCNT-3 maintained the characteristic of preferential killing of HCT116 over IMR-90 (Figure 8C). Nevertheless, compared with mere CDDP treatment, our construct was poorer in reducing cancer cell survival. HCT116 cell survival was only reduced significantly to approximately $30 \%$ with highest concentration of CDDP@f-MWCNT-3 treatment. The poorer performance of CDDP delivered by our MWCNT-based nanocarrier could be accounted by possible slow removal of AuNPs caps in cells, which in turn impede rapid release of CDDP for targeted cytotoxic action. Our reasoning is partially supported by the cytotoxicity profile of CDDP delivered by uncapped MWCNT-OX: CDDP@MWCNT-OX exhibited desirable dose-dependent cytotoxicity in HCT116 cells, despite being less efficacious than free CDDP treatment (Figure 8D versus Figure $8 \mathrm{~A}$ ). This infers that, in addition to being capable in delaying CDDP leakage, AuNPs might actually impede controlled release of CDDP despite the presence of triggering agents. As a result, optimization of alternative capping strategies is warranted for construction for efficacious MWCNTbased delivery system for CDDP.

Notably, in contrast to AuNP-capped MWCNTs through disulfide linkage, MWCNT-OX did not render selectivity in cytotoxicity against tumorous cells. Similar dose-dependent cytotoxicity to HCT116 cells was also observed in non-malignant IMR-90 cells (Figure 8D). Hence, we suggest that MWCNTs efficiency in permeating into intracellular space is indifferent for cancerous and normal cells. Moreover, our findings indicate that the use of disulfide linkage in MWCNT-based drug delivery system indeed conferred slight selectivity toward cancerous cells. As a result, it would be of great interest to consider the use of either targeting groups or specific chemical linkages in engineering smart delivery systems with the objective of eradicating cancerous cell selectively. 

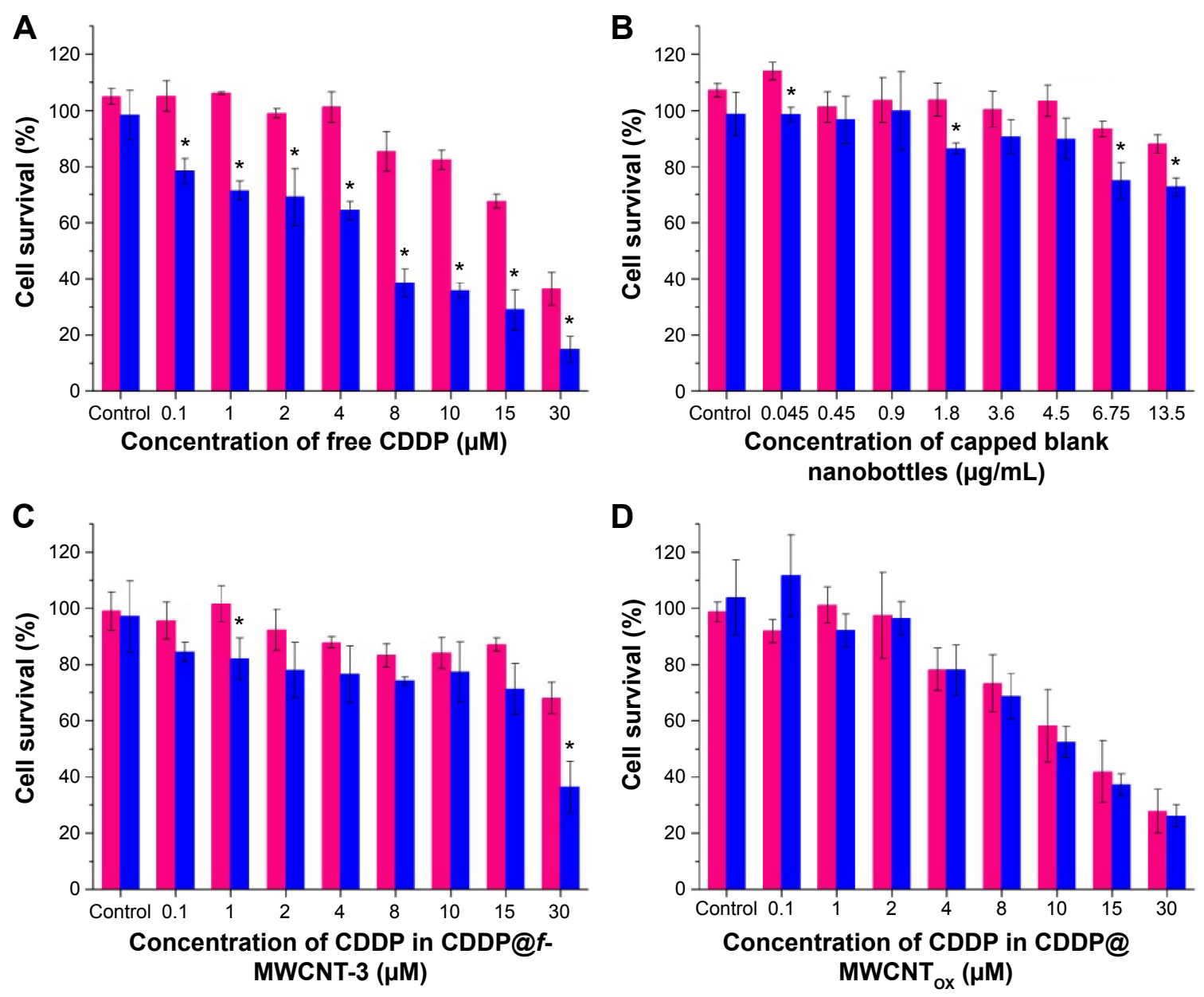

IMR-90 HCT-116

Figure 8 Cell viability of IMR-90 and HCT-II6 treated with (A) free CDDP; (B) capped blank nanobottles without CDDP; (C) capped nanobottles CDDP@f-MWCNT-3 with CDDP inside; and (D) uncapped MWCNT ox - CDDP, respectively. $* P<0.05$ compared with IMR-90 (Student's $t$-test).

Abbreviations: CDDP, cis-diammineplatinum(II) dichloride; DTT, dithiothreitol; MWCNT, multi-walled carbon nanotube; PBS, phosphate-buffered saline.

In addition, it should be noted that this construct, made up of GSH-sensitive bonds and AuNPs, could be additionally exploited in hyperthermia applications, whereby the AuNPs could provide further destruction of the selected cancer cells upon exposure to an external electromagnetic field. ${ }^{50}$

We also investigated cell viability of HCT116 cells and IMR-90 cells upon incubation with AuNP-capped MWCNTs without CDDP (Figure 8B). AuNP-capped MWCNTs blank nanobottles displayed marginal impact to cell survival at the higher tested concentrations $(6.75$ and $13.5 \mu \mathrm{g} / \mathrm{mL}$ ). Therefore, the cytotoxic effect observed with CDDP loaded constructs could be primarily attributed to the activity of encapsulated CDDP instead of MWCNT nanocarrier.

\section{Conclusion}

A novel "nanobottle" drug delivery system was engineered by capping AuNPs to the open-tips of MWCNTs via different cleavable linkage containing linkers, namely hydrazone, ester, and disulfide linkers. High-resolution imaging of the constructs by TEM revealed the presence of AuNPs at extremities of MWCNTs, indicating successful covalent linkage of AuNPs to MWCNTs open-tips via defect functionalization. In vitro release studies showed that only CDDP@fMWCNT-3, which contains disulfide linkage, demonstrated intended controlled release of CDDP in the presence of the reducing agent DTT. CDDP@f-MWCNT-3 was found to exert stronger cytotoxic effect in cancerous HCT116 cells than in normal IMR-90 cells, highlighting the preservation of CDDP selectivity in GSH-overexpressing cancer cells after encapsulation in MWCNTs. Therefore, by tuning capping strategies or introducing targeting groups, a smart MWCNT-based delivery system that combines selective bond-cleavage and eventually localized hyperthermia could be devised for efficient CDDP delivery to enhance current therapeutic anticancer strategies. 


\section{Acknowledgments}

This research has been supported by the National University of Singapore, Department of Pharmacy ((AcRF) Tier 1-FRC grant R-148-000-164-112 and NUSAGE N-148-000-009-001) and by Ministry of Education of Singapore (grant MOE2011T21-1201-P09; R-144-000-306-112). NUS Graduate School for Integrative Sciences and Engineering graduate fellowship is thankfully acknowledged by SLY, WJG, MD, and AP for financial support of their graduate studies.

\section{Disclosure}

The authors report no conflicts of interest in this work.

\section{References}

1. Leyva-García S, Morallón E, Cazorla-Amorós D, Béguin F, LozanoCastelló D. New insights on electrochemical hydrogen storage in nanoporous carbons by in situ Raman spectroscopy. Carbon. 2014;69: 401-408.

2. Furukawa H, Yaghi OM. Storage of hydrogen, methane, and carbon dioxide in highly porous covalent organic frameworks for clean energy applications. J Am Chem Soc. 2009;131:8875-8883.

3. McKeown NB, Gahnem B, Msayib KJ, et al. Towards polymer-based hydrogen storage materials: engineering ultramicroporous cavities within polymers of intrinsic microporosity. Angew Chem Int Ed Engl. 2006;45:1804-1807.

4. Oh JM, Park DH, Choi SJ, Choy JH. LDH nanocontainers as bioreservoirs and drug delivery carriers. Recent Pat Nanotechnol. 2012; 6:200-217.

5. Goldberg M, Langer R, Jia X. Nanostructured materials for applications in drug delivery and tissue engineering. J Biomater Sci Polym Ed. 2007; $18: 241-268$

6. Chang FP, Hung Y, Chang JH, Lin CH, Mou CY. Enzyme encapsulated hollow silica nanospheres for intracellular biocatalysis. ACS Appl Mater Interfaces. 2014;6:6883-6890.

7. Du X, Sun Y, Tan B, et al. Troger's base-functionalised organic nanoporous polymer for heterogeneous catalysis. Chem Commun (Camb). 2010;46:970-972.

8. Dang D, Wu P, He C, Xie Z, Duan C. Homochiral metal-organic frameworks for heterogeneous asymmetric catalysis. $\mathrm{Am}$ Chem Soc. 2010;132:14321-14323.

9. McKeown NB, Budd PM. Polymers of intrinsic microporosity (PIMs): organic materials for membrane separations, heterogeneous catalysis and hydrogen storage. Chem Soc Rev. 2006;35:675-683.

10. Savic R, Luo L, Eisenberg A, Maysinger D. Micellar nanocontainers distribute to defined cytoplasmic organelles. Science. 2003;300: 615-618.

11. Zhao Y, Burkert SC, Tang Y, et al. Nano-gold corking and enzymatic uncorking of carbon nanotube cups. J Am Chem Soc. 2015;137: 675-684.

12. Vriezema DM, Comellas Aragones M, Elemans JA, Cornelissen JJ, Rowan AE, Nolte RJ. Self-assembled nanoreactors. Chem Rev. 2005; 105:1445-1489.

13. Ajima K, Yudasaka M, Murakami T, Maigné A, Shiba K, Iijima S. Carbon nanohorns as anticancer drug carriers. Mol Pharm. 2005;2: 475-480.

14. Ren Y, Pastorin G. Incorporation of hexamethylmelamine inside capped carbon nanotubes. Adv Mater. 2008;20:2031-2036.

15. Worsdorfer B, Pianowski Z, Hilvert D. Efficient in vitro encapsulation of protein cargo by an engineered protein container. J Am Chem Soc. 2012; 134:909-911.

16. Jin $\mathrm{Y}$, Gao X. Spectrally tunable leakage-free gold nanocontainers. J Am Chem Soc. 2009;131:17774-17776.
17. Leung KCF, Nguyen TD, Stoddart JF, Zink JI. Supramolecular nanovalves controlled by proton abstraction and competitive binding. Chem Mater. 2006;18:5919-5928.

18. Kondyurin A, Levchenko I, Han ZJ, et al. Hybrid graphite film-carbon nanotube platform for enzyme immobilization and protection. Carbon. 2013;65:287-295

19. Park C, Oh K, Lee SC, Kim C. Controlled release of guest molecules from mesoporous silica particles based on a $\mathrm{pH}$-responsive polypseudorotaxane motif. Angew Chem Int Ed Engl. 2007;46:1455-1457.

20. Han Y, Shchukin D, Fernandes P, Mutihac R-C, Mohwald H. Mechanism and kinetics of controlled drug release by temperature stimuli responsive protein nanocontainers. Soft Matter. 2010;6:4942-4947.

21. Nguyen TD, Leung KCF, Liong M, Liu Y, Stoddart JF, Zink JI. Versatile supramolecular nanovalves reconfigured for light activation. Adv Funct Mater. 2007;17:2101-2110.

22. Park C, Kim H, Kim S, Kim C. Enzyme responsive nanocontainers with cyclodextrin gatekeepers and synergistic effects in release of guests. J Am Chem Soc. 2009;131:16614-16615.

23. Nguyen TD, Liu Y, Saha S, Leung KC, Stoddart JF, Zink JI. Design and optimization of molecular nanovalves based on redox-switchable bistable rotaxanes. J Am Chem Soc. 2007;129:626-634.

24. Vashist SK, Zheng D, Pastorin G, Al-Rubeaan K, Luong JHT, Sheu F-S. Delivery of drugs and biomolecules using carbon nanotubes. Carbon. 2011;49:4077-4097.

25. Stan G, Cole MW. Low coverage adsorption in cylindrical pores. Surf Sci. 1998;395:280-291.

26. Simonyan VV, Johnson JK, Kuznetsova A, Yates JT. Molecular simulation of xenon adsorption on single-walled carbon nanotubes. J Chem Phys. 2001;114:4180-4185.

27. Rawat DS, Heroux L, Krungleviciute V, Migone AD. Adsorption of xenon on purified HiPco single walled carbon nanotubes. Langmuir. 2006;22:234-238

28. Murakami T, Ajima K, Miyawaki J, Yudasaka M, Iijima S, Shiba K. Drug-loaded carbon nanohorns: adsorption and release of dexamethasone in vitro. Mol Pharm. 2004;1:399-405.

29. Ajima K, Yudasaka M, Maigné A, Miyawaki J, Iijima S. Effect of functional groups at hole edges on cisplatin release from inside single-wall carbon nanohorns. J Phys Chem B. 2006;110:5773-5778.

30. Tripisciano C, Kraemer K, Taylor A, Borowiak-Palen E. Single-wall carbon nanotubes based anticancer drug delivery system. Chem Phys Lett. 2009;478:200-205.

31. Guven A, Rusakova IA, Lewis MT, Wilson LJ. Cisplatin@UStube carbon nanocapsules for enhanced chemotherapeutic delivery. Biomaterials. 2012;33:1455-1461.

32. Li J, Yap SQ, Yoong SL, et al. Carbon nanotube bottles for incorporation, release and enhanced cytotoxic effect of cisplatin. Carbon. 2012; 50:1625-1634.

33. Wong SS, Joselevich E, Woolley AT, Cheung CL, Lieber CM. Covalently functionalized nanotubes as nanometre- sized probes in chemistry and biology. Nature. 1998;394:52-55.

34. Wong SS, Woolley AT, Joselevich E, Cheung CL, Lieber CM. Covalently-functionalized single-walled carbon nanotube probe tips for chemical force microscopy. J Am Chem Soc. 1998;120:8557-8558.

35. Ali-Boucetta H, Al-Jamal KT, Müller KH, et al. Cellular uptake and cytotoxic impact of chemically functionalized and polymer-coated carbon nanotubes. Small. 2011;7:3230-3238.

36. Danhier F, Feron O, Preat V. To exploit the tumor microenvironment: passive and active tumor targeting of nanocarriers for anti-cancer drug delivery. J Control Release. 2010;148:135-146.

37. Zhao Y, Tang Y, Chen Y, Star A. Corking carbon nanotube cups with gold nanoparticles. ACS Nano. 2012;6:6912-6921.

38. Wu W, Wieckowski S, Pastorin G, Briand JP, Prato M, Bianco A. Targeted delivery of amphotericin B to cells using functionalised carbon nanotubes. Angew Chem Int Ed. 2005;44:6358-6362.

39. Muzi L, Ménard-Moyon C, Russier J, et al. Diameter-dependent release of a cisplatin pro-drug from small and large functionalized carbon nanotubes. Nanoscale. 2015;7(12):5383-5394. 
40. Hruby M, Konak C, Ulbrich K. Polymeric micellar $\mathrm{pH}$-sensitive drug delivery system for doxorubicin. J Control Release. 2005;103: 137-148.

41. Oh JE, Nam YS, Lee KH, Park TG. Conjugation of drug to poly(D,Llactic-co-glycolic acid) for controlled release from biodegradable microspheres. J Control Release. 1999;57:269-280.

42. Meng F, Hennink WE, Zhong Z. Reduction-sensitive polymers and bioconjugates for biomedical applications. Biomaterials. 2009;30: 2180-2198.

43. Chang TC, Chang MJ, Hsueh S. Glutathione concentration and distribution in cervical cancers and adjacent normal tissues. Gynecol Obstet Invest. 1993;36:52-55.

44. Perry RR, Mazetta JA, Levin M, Barranco SC. Glutathione levels and variability in breast tumors and normal tissue. Cancer. 1993;72: 783-787.
45. Wong DY, Hsiao YL, Poon CK, et al. Glutathione concentration in oral cancer tissues. Cancer Lett. 1994;81:111-116.

46. Gilbert HF. Molecular and cellular aspects of thiol disulfide exchange. Adv Enzymol Relat Areas Mol Biol. 1990;63:69-172.

47. Gilbert HF. Thiol/disulfide exchange equilibria and disulfide bond stability. Methods Enzymol. 1995;251:8-28.

48. Chen XC. Carbon-nanotube-based stimuli-responsive controlled-release system. Chemistry. 2011;17(16):4454-4459.

49. Ishimoto T, Nagano O, Yae T, et al. CD44 variant regulates redox status in cancer cells by stabilizing the $\mathrm{xCT}$ subunit of system $\mathrm{xc}^{-}$-and thereby promotes tumor growth. Cancer Cell. 2011;19:387-400.

50. Cherukuri P, Glazer ES, Curley SA. Targeted hyperthermia using metal nanoparticles. Adv Drug Deliv Rev. 2010;62(3):339-345. 


\section{Supplementary materials}

Table SI Concentrations of CDDP, CDDP@f-MWCNT-3, CDDP@MWCNT-OX, and blank disulfide bond nanobottles used for the cell treatment

\begin{tabular}{lllll}
\hline $\begin{array}{l}\text { Molar concentration } \\
(\mu \mathrm{M})\end{array}$ & $\begin{array}{l}\text { CDDP } \\
(\mu \mathrm{g} / \mathrm{mL})\end{array}$ & $\begin{array}{l}\text { CDDP@f-MWCNT-3 } \\
(\mu \mathrm{g} / \mathrm{mL})\end{array}$ & $\begin{array}{l}\text { Blank capped nanobottles } \\
(\mu \mathrm{g} / \mathrm{mL})\end{array}$ & $\begin{array}{l}\text { CDDP@MWCNT-OX } \\
(\mu \mathrm{g} / \mathrm{mL})\end{array}$ \\
\hline 0.1 & 0.03 & 0.075 & 0.045 & 0.05 \\
1 & 0.3 & 0.75 & 0.45 & 0.51 \\
2 & 0.6 & 1.5 & 0.9 & 1.02 \\
4 & 1.2 & 3 & 1.8 & 2.03 \\
8 & 2.4 & 6 & 3.6 & 4.07 \\
10 & 3 & 7.5 & 4.5 & 5.08 \\
15 & 4.5 & 11.25 & 6.75 & 7.63 \\
30 & 9 & 22.5 & 13.5 & 15.25
\end{tabular}

Notes: The concentrations of CDDP and nanotubes were comparable to the corresponding concentration of CDDP and nanotubes in CDDP@f-MWCNT-3. The molar concentration is the molar concentration of CDDP.

Abbreviations: CDDP, cis-diammineplatinum(II) dichloride; MWCNT, multi-walled carbon nanotube.

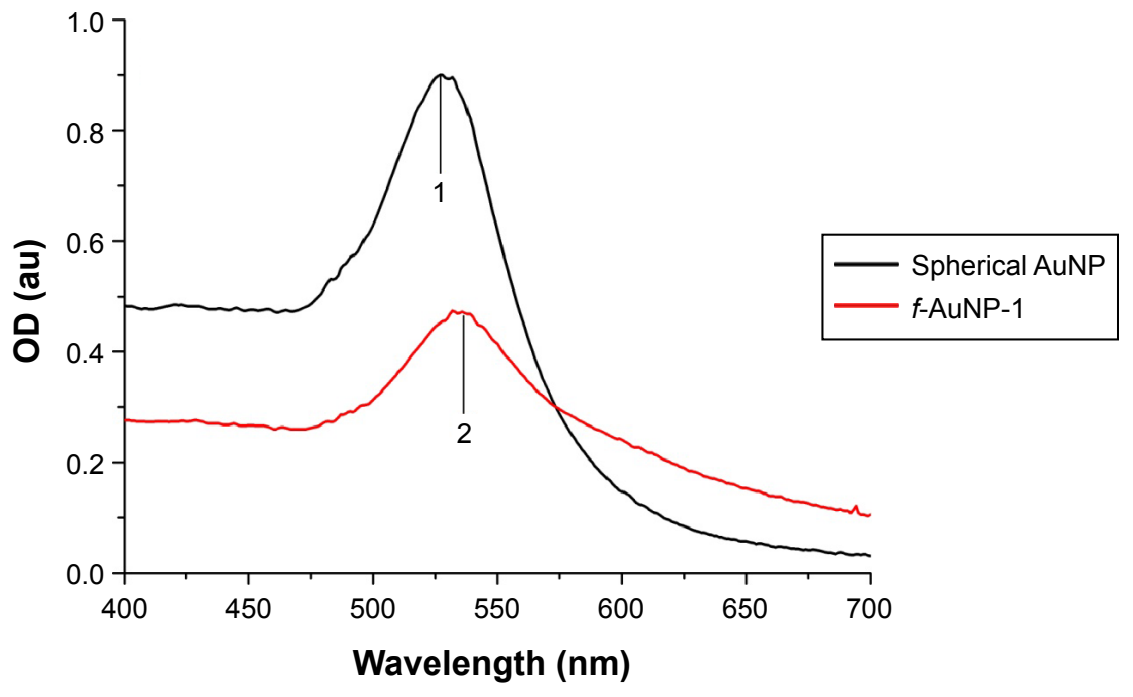

Figure SI UV-vis spectra of spherical AuNP (black line) and f-AuNP-I (red line). $\lambda_{\max }:$ I $-527 \mathrm{~nm}$; $2-536 \mathrm{~nm}$. Abbreviations: AuNP, gold nanoparticle; OD, optical density. 

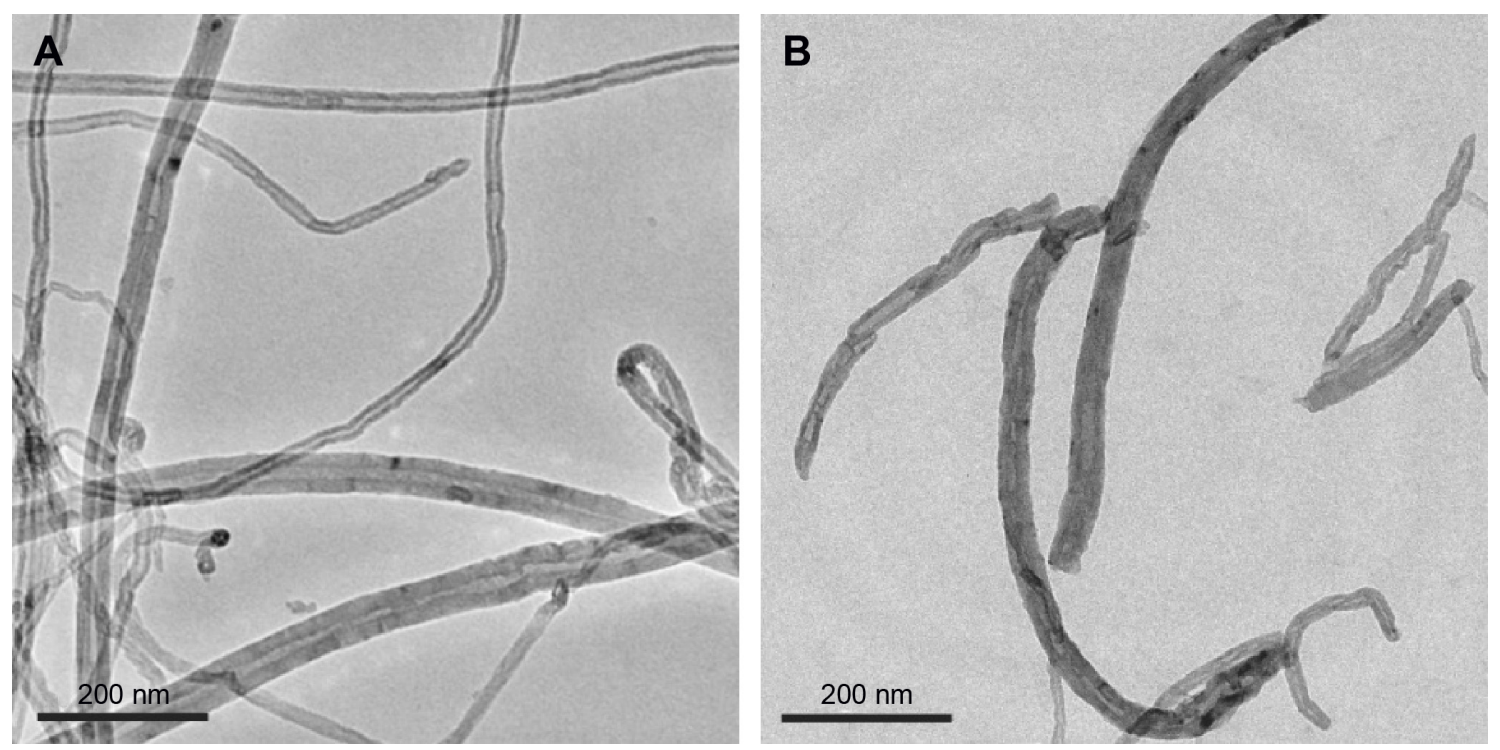

Figure S2 TEM images of (A) pristine MWCNT and (B) MWCNT-OX.

Abbreviations: TEM, transmission electron microscopy; MWCNT, multi-walled carbon nanotubes.
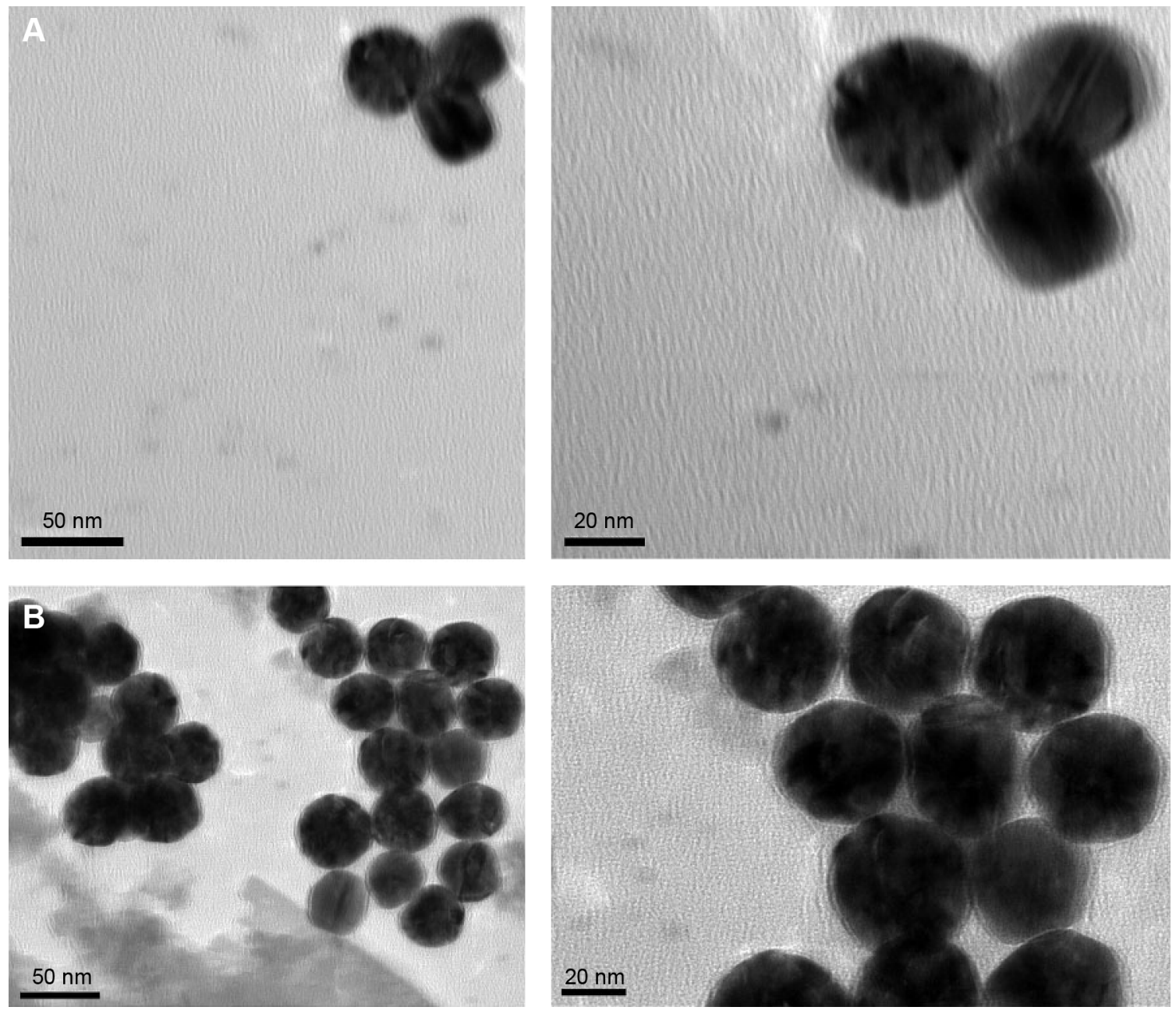

Figure S3 TEM images of $f$-AuNP-2 (A) and f-AuNP-3 (B).

Abbreviations: TEM, transmission electron microscopy; AuNP, gold nanoparticles. 


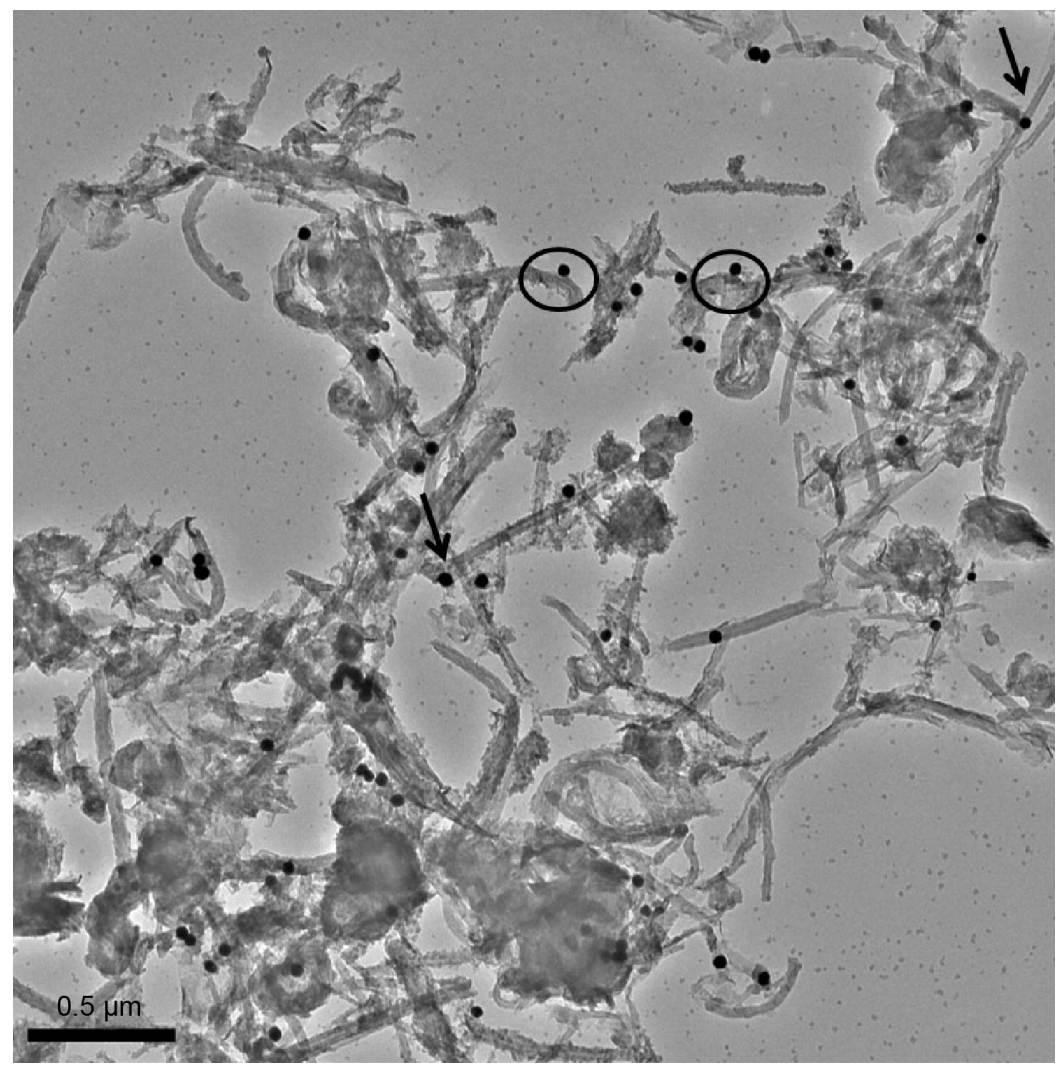

Figure S4 TEM images of $f$-MWCNT-I, with AuNPs at the tips (as indicated by arrows) or at the side-wall (in circle)

Abbreviations: TEM, transmission electron microscopy; MWCNT, multi-walled carbon nanotubes; AuNP, gold nanoparticle.
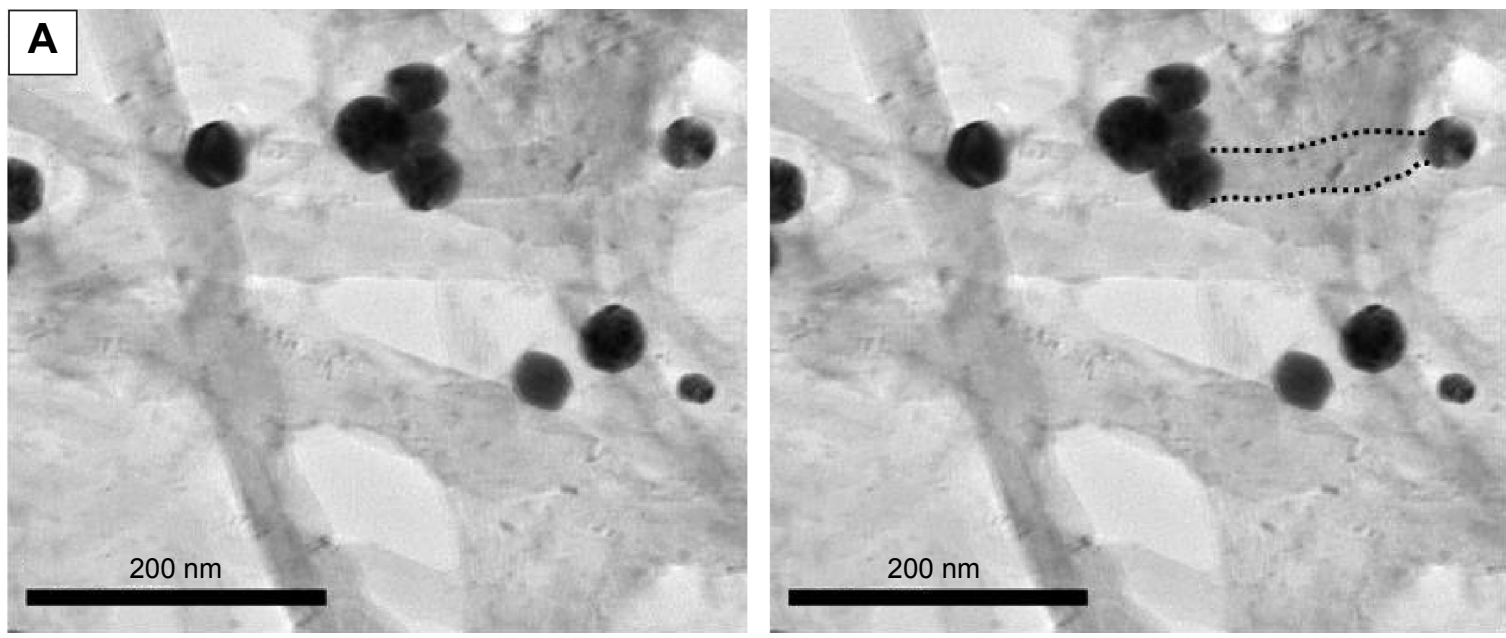

Figure S5 (Continued) 

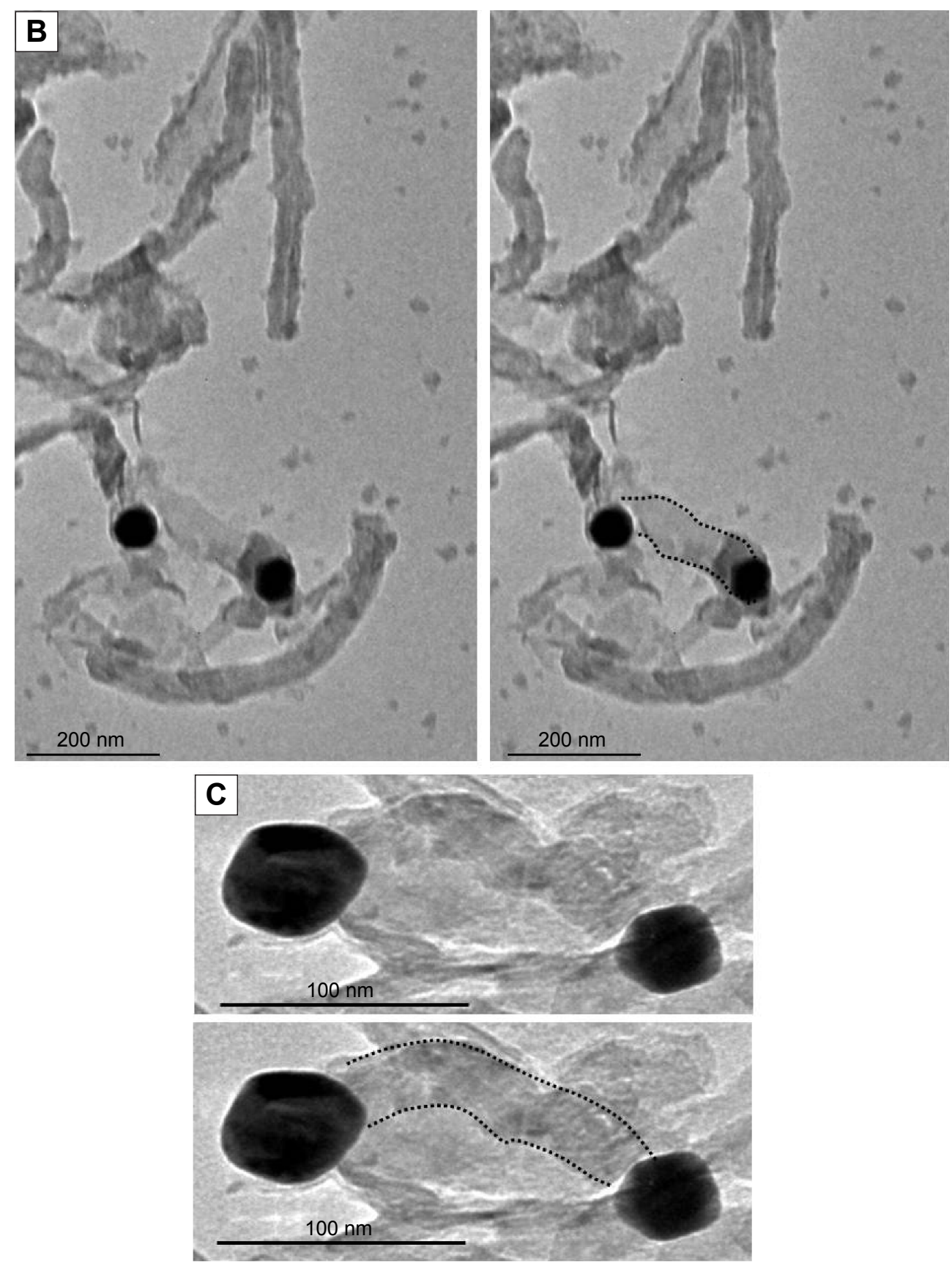

Figure S5 TEM images of MWCNT+ AuNPs linked through cleavable bonds.

Notes: Dashed lines are added to highlight the tube's structure. (A) f-MWCNT-3. (B) f-MWCNT-2. (C) f-MWCNT-I.

Abbreviations: TEM, transmission electron microscopy; MWCNT, multi-walled carbon nanotubes; AuNP, gold nanoparticle.

International Journal of Nanomedicine

\section{Publish your work in this journal}

The International Journal of Nanomedicine is an international, peerreviewed journal focusing on the application of nanotechnology in diagnostics, therapeutics, and drug delivery systems throughout the biomedical field. This journal is indexed on PubMed Central, MedLine, CAS, SciSearch ${ }^{\circledR}$, Current Contents ${ }^{\circledR} /$ Clinical Medicine,

\section{Dovepress}

Journal Citation Reports/Science Edition, EMBase, Scopus and the Elsevier Bibliographic databases. The manuscript management system is completely online and includes a very quick and fair peer-review system, which is all easy to use. Visit http://www.dovepress.com/ testimonials.php to read real quotes from published authors.

\footnotetext{
Submit your manuscript here: http://www.dovepress.com/international-journal-of-nanomedicine-journal
} 